\title{
TWO REMARKABLE EXAMPLES OF CONAL PROFILE EVOLUTION
}

\author{
T. H. HANKINS \\ Very Large Array, National Radio Astronomy Observatory \\ and Department of Physics, New Mexico Institute of Mining and Technology \\ JOANNA M. RANKIN \\ Department of Physics, University of Vermont \\ D. R. STINEbring \\ Department of Physics, Oberlin College \\ M. M. MCKINNON \\ Very Large Array, National Radio Astronomy Observatory \\ and Department of Physics, New Mexico Institute of Mining and Technology
}

\begin{abstract}
Pulsar 0301+19, whose two well-separated components provide a textbook example of the conal double profile, apparently evolves to a single form at frequencies above $5 \mathrm{GHz}$. PSR $2016+28$, by contrast, exhibits a single form above $200 \mathrm{MHz}$, but bifurcates strongly and very asymmetrically below $100 \mathrm{MHz}$. In few pulsars can we see the full possibilities of conal profile evolution so clearly manifested.
\end{abstract}

\section{Introduction}

Two principal types of conal profiles are known (Rankin 1983a, Rankin 1983b, Rankin 1986), the conal single $\left(\mathbf{S}_{\mathrm{d}}\right)$ and the conal double $(\mathrm{D})$. Both types of profile are produced by a traverse of the sight line through a hollow-conical emission zone. This zone is closely associated with the bundle of field lines immediately surrounding the magnetic pole of the neutron star; see figure 1 .

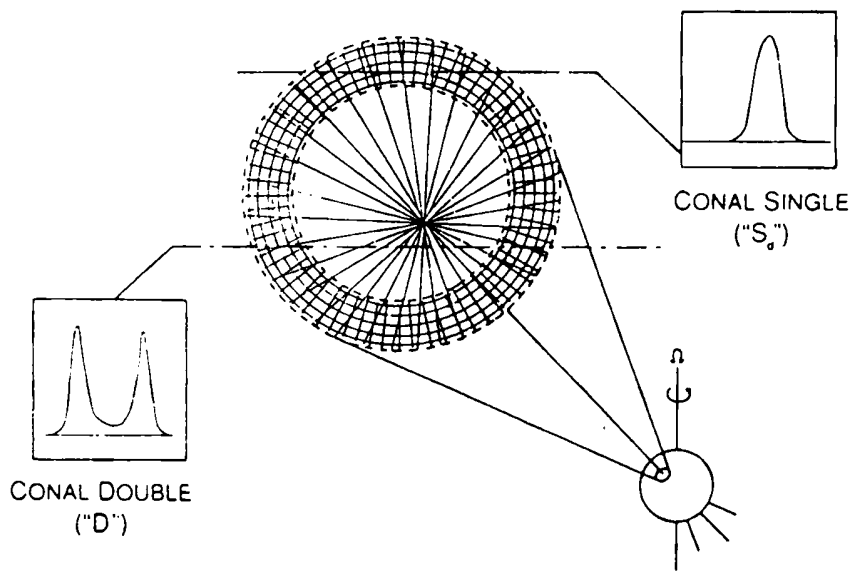

Figure 1 Schematic diagram showing the geometry of conal beaming.

No physical distinction can be drawn between these two types of profile; they differ only in sightline geometry. In the case of the double profile, the line of sight makes a central traverse through the conical emission zone, whereas for the conal single profile, the sight line makes a tangential traverse,



Figure 2 A set of multifrequency profiles for the pulsar $0301+19$ aligned absolutely in time using the dispersion measure value, $D M=16.50 \mathrm{pc} \mathrm{cm}^{-3}$.

that is, one that only just grazes the edge of the emission zone. 


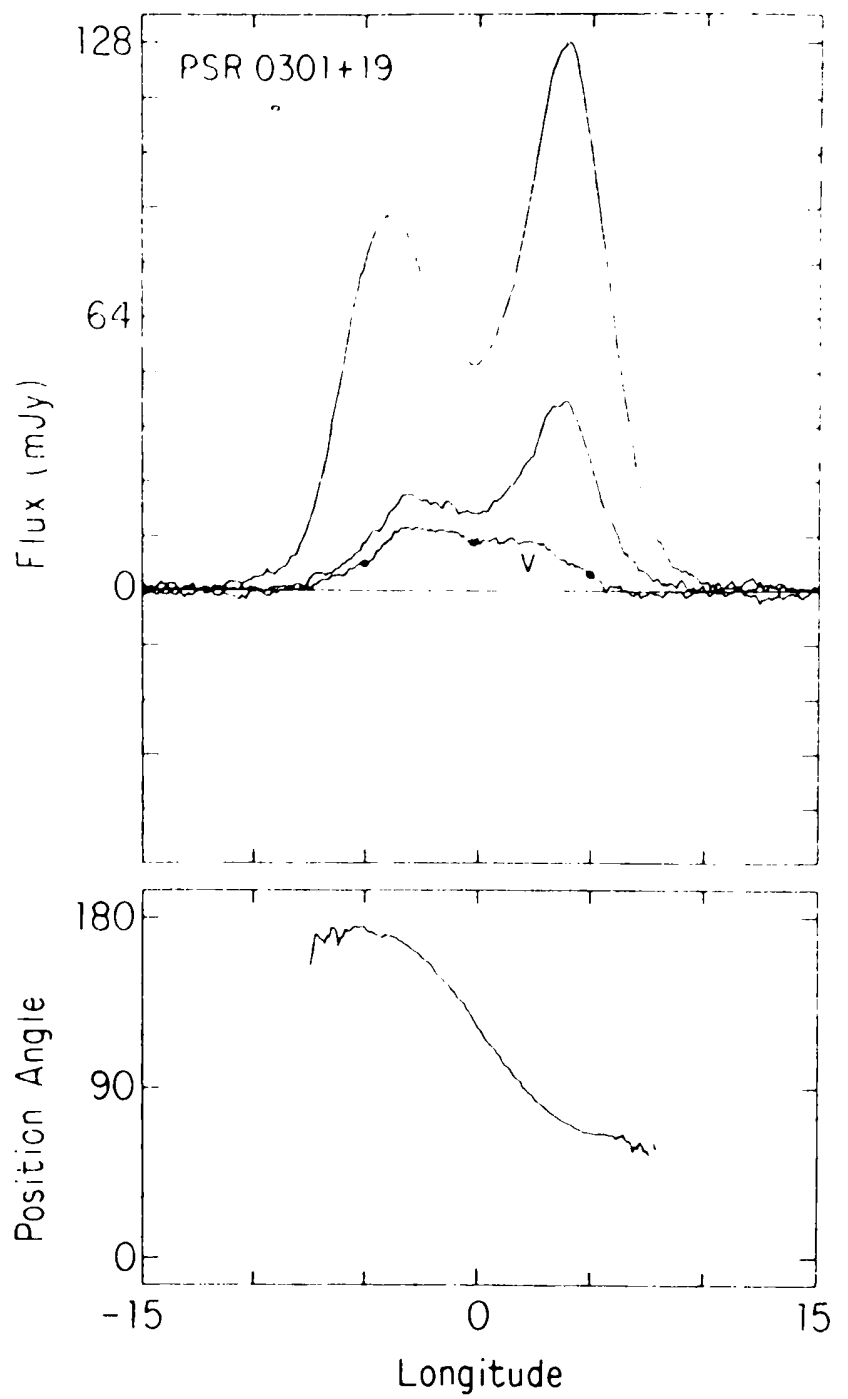

Figure 3 1400-M Hz average polarization profile for pulsar $0301+19$ (from Rankin, Stinebring and Weisberg 1989).

The emission cone becomes larger at lower frequencies probably because the lower frequency emission is produced at progressively greater heights in the magnetosphere as the bundle of dipolar field lines opens outward (with width, $W$, scaling approximately as $\left.f^{-0.25}\right)$. Therefore, at lower frequencies conal single $\left(\mathbf{S}_{\mathrm{d}}\right)$ profiles tend to bifurcate and the components of double (D) profiles become progressively more widely separated.

\section{PSR 0301+19}

This pulsar provides one of the best studied and clearest examples of the (conal) double profile (D) class. Its evolution with frequency is shown in figure 2 , and its other characteristics include:

- two well separated components, whose separation increases progressively at low frequencies due to the spreading of the hollow-conical emission region.
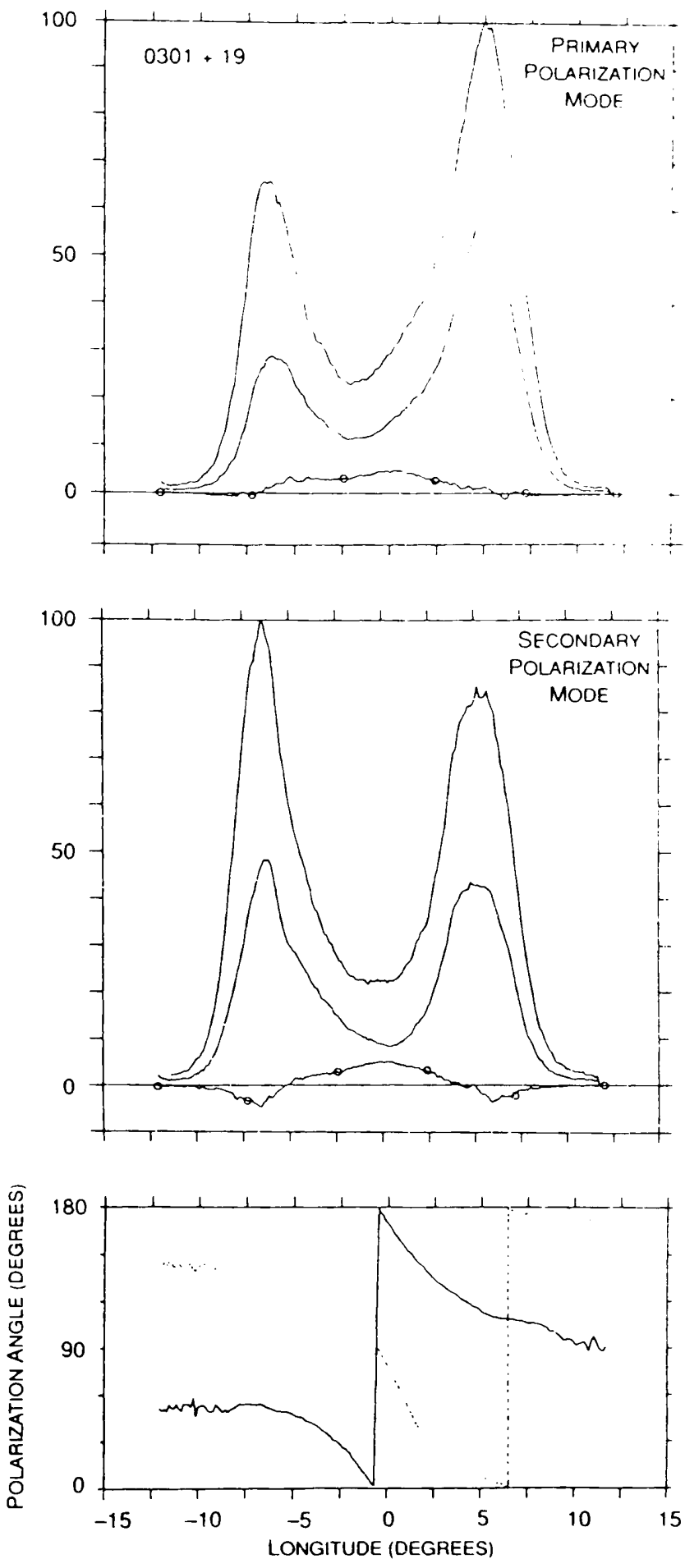

Figure 4 Average $430-\mathrm{MHz}$ polarization profiles for pulsar $0301+19$ in which the two orthogonal polarization modes have been separated (see Rankin 1988). The profiles contain some 635 and 552 effective pulses and have scale intensities of 14.4 and $12.0 \mathrm{Jy}$, respectively.

- a $\leq 180^{\circ}$ "S"-shaped traverse of the linear polarization angle, indicating that the sight line to the pulsar cuts the hollow-conical emission zone centrally as shown in figure 3.

- orderly subpulse modulation associated with the two principal components, showing sor 




Figure 5 A set of multifrequency profiles for pulsar $2016+28$ aligned absolutely in time using the dispersion measure value, $D M=14.196 \mathrm{pc} \mathrm{cm}^{-3}$.

actual subpulse motion or drifting indicating that the sight line cuts the conal emission zone somewhat tangentially.

- depolarization on the outer edges of the components caused by the "orthogonal" polarization modes. Compare figure 4.

- pulse nulling.

Pulsar 0301+19 exhibits all of these standard characteristics of conal double profiles. Note, however, in figure 2 that the $6 \mathrm{~cm}(4880 \mathrm{MHz})$ observation of the profile is single! This is a very unusual pattern of evolution for a conal double profile at high frequency, but it is entirely compatible and understandable in terms of the basic conal emission geometry involved.

\section{PSR 2016+28}

This pulsar represents one of the best examples of the conal single profile $\left(S_{d}\right)$ species. The evolution of its profile with frequency is given in figure 5 , and furthermore it has:
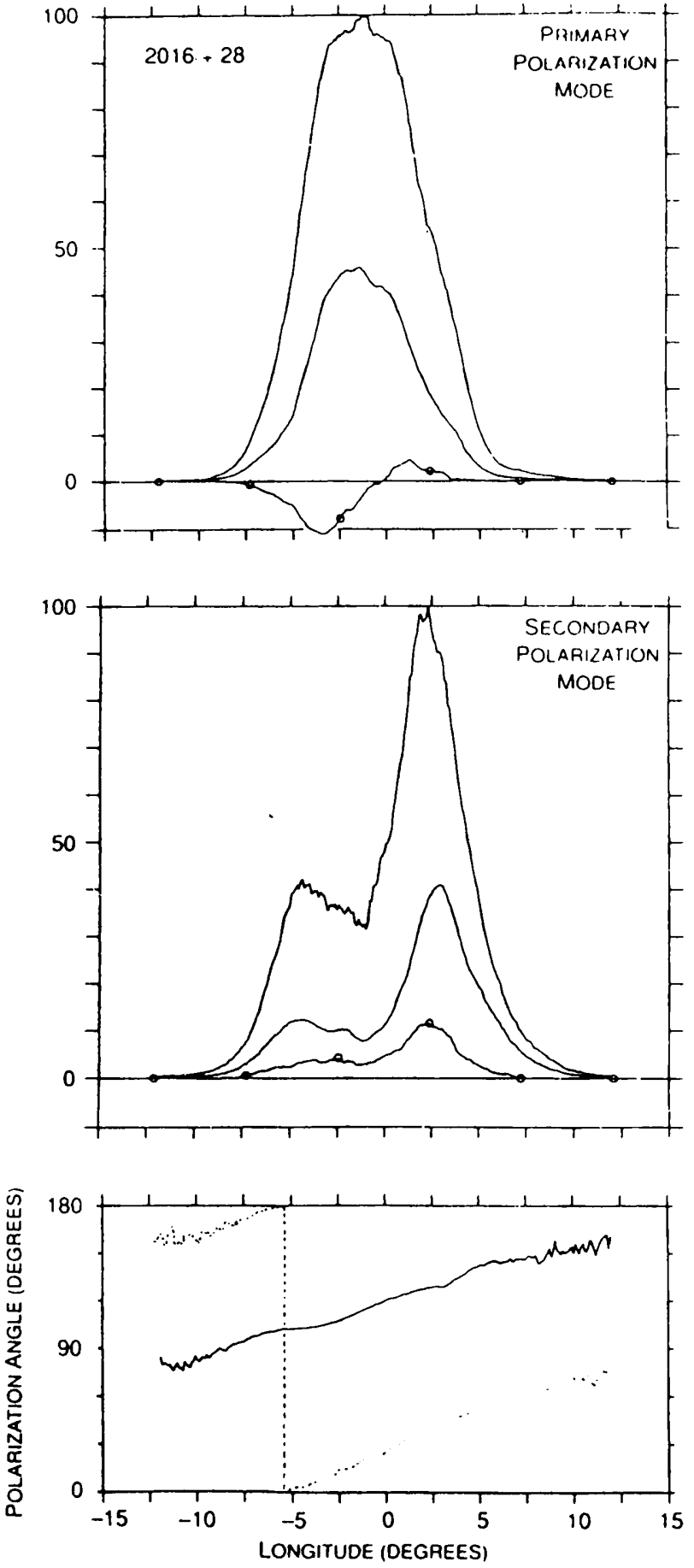

Figure $61400-\mathrm{MHz}$ average polarization profile for pulsar 2016+28 (from Stinebring et al. 1984).

- a single component, whose width increases progressively at low frequencies, due to the spreading of the hollow-conical emission region.

- a shallow intrinsic traverse of the linear polarization angle, indicating that the sight line to the pulsar cuts the hollow-conical emission zone tangentially as shown in figures 6 and 7.

- orderly drifting subpulses over the entire width of the profile, reiterating that the sight 




Figure 7 Histogram of the distribution of polarization for pulsar 2016+28 (from Stinebring et al. 1984).

line cuts the conal emission zone tangentially.

- a prominent $45^{\circ}$ "jump" and depolarization on the outer edges of the profile due to the "orthogonal" polarization modes. See figures 7 and 8.

Pulsar 2016+28 exhibits all of these regular characteristics of conal single profiles. Over most of the spectrum its profile is single, though at meter wavelengths it might best be described as an "unresolved double". However, below about $200 \mathrm{MHz}$, the profile bifurcates strongly, and the trailing component becomes progressively weaker than the leading feature.

Such steep conal spreading below $100 \mathrm{MHz}$ is certainly unusual, but again, it is entirely understandable on the basis of the basic geometry of conal emission. In addition, the low frequency asymmetry may well be related to the "absorption" phenomenon.
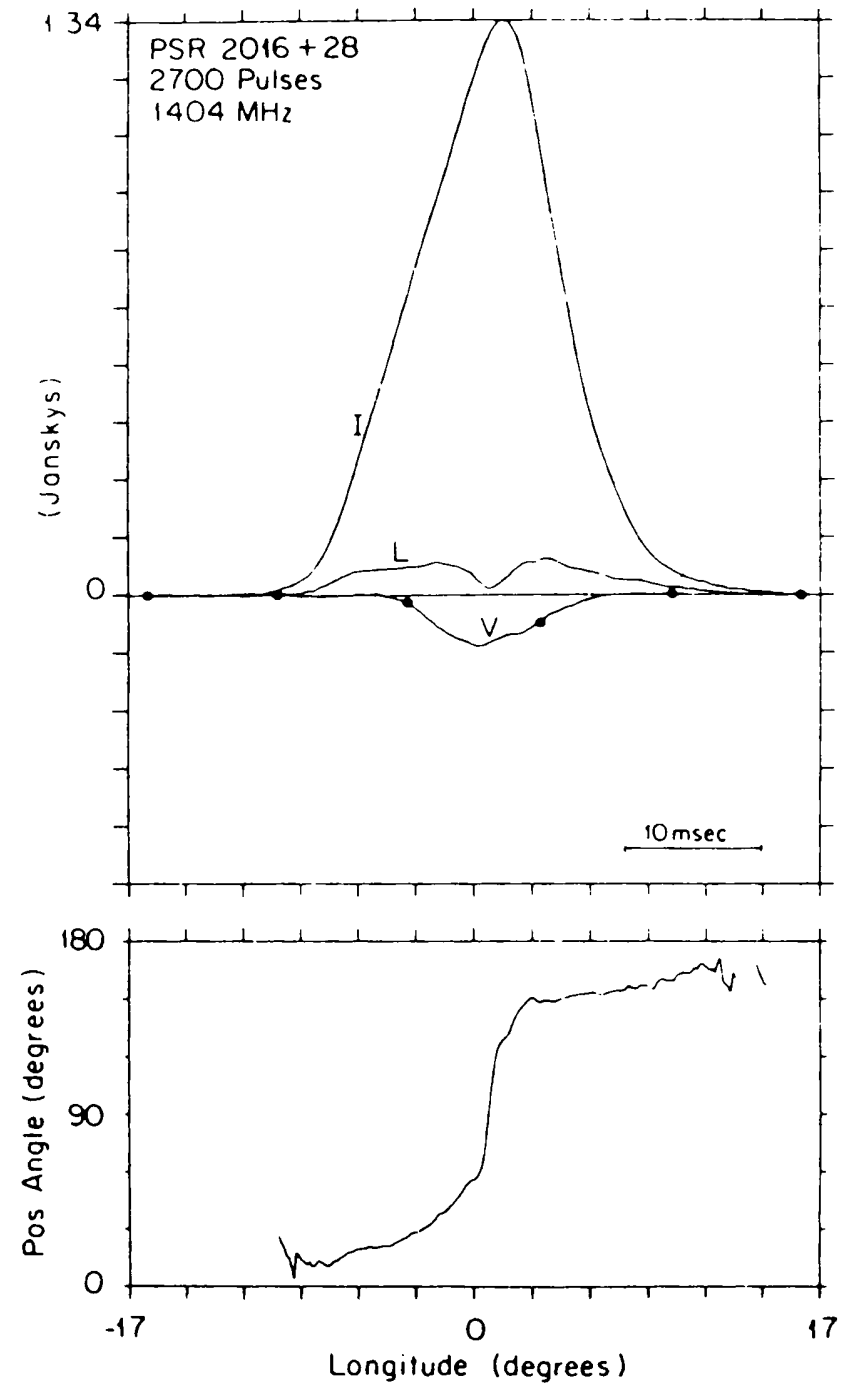

Figure 8 Average $430-\mathrm{MHz}$ polarization profiles for the pulsar $2016+28$ in which the two orthogonal polarization modes have been separated (see Rankin 1988). The profiles contain some 620 and 653 effective pulses and have scale intensities of 269.3 and $119.6 \mathrm{Jy}$, respectively. 